\title{
Estratificación digital: acceso y usos de las TIC en la población escolar chilena
}

\section{Digital Stratification: ICT Access and Use in Chilean Students}

\author{
Carlos Rodríguez Garcés (*) carlosro@ubiobio.cl \\ Daniela Sandoval Muñoz (*) dfsandov@ubiobio.cl \\ (*) Universidad del Bío Bío \\ (Recibido: 24 de septiembre de 2014; Aceptado para su publicación: 30 de octubre de 2015)
}

Cómo citar: Rodríguez, C. y Sandoval, D. (2017). Estratificación digital: acceso y usos de las Tic en la población escolar de Chile. Revista Electrónica de Investigación Educativa, 19(1), 21-34. Recuperado de https://doi.org/10.24320/redie.2017.19.1.902

\section{Resumen}

Las TIC ocupan en la actualidad un importante espacio en la vida cotidiana, pues son utilizadas en diversos contextos y con diferentes finalidades; no obstante, existe un sector de la población excluido de las oportunidades que las TIC brindan, exclusión determinada por el nivel socioeconómico. Utilizando bases de datos nacionales, en la presente investigación se hace un análisis descriptivo-correlacional de la penetración y usos de las TIC en la población escolar de enseñanza básica y media en Chile. Los resultados indican que el acceso a Internet y a las tecnologías está fuertemente segmentado; la estratificación digital va más allá del acceso y existen usos diferenciados con base en el capital cultural del estudiante. La escuela se configura como el principal elemento compensador de la brecha digital posibilitando el acceso (aunque restringido) a las TIC para una proporción importante de estudiantes de los sectores de menores ingresos.

Palabras clave: TIC, brecha digital, capital cultural, nivel socioeconómico.

\begin{abstract}
ICT plays an increasingly prominent role in daily life, and is used in varying contexts to fulfill different objectives, but one sector of the population is denied the opportunities offered by ICT; this exclusion is dictated by socio-economic status. This study draws on national databases to provide a descriptive-correlational analysis of ICT adoption and use in the student population in primary and secondary education. The results show that Internet and technology access is strongly segmented; digital stratification goes beyond access, and different uses are observed based on the student's cultural capital. Schools are most successful at offsetting the digital divide, making access to ICT possible (albeit restricted) for a significant number of students in lower-income sectors.
\end{abstract}


Keywords: ICT, digital gap, digital stratification, cultural capital, technological access.

\section{Introducción}

El término "usuario", o su homólogo "internauta", es un concepto polisémico y complejo, difícil de medir, puesto que además del simple acceso implica antigüedad o permanencia, frecuencia de uso, tipos de servicios y contenidos utilizados, entre otros factores discriminantes de relevancia. En consecuencia, no trata exclusivamente sobre el acceso, como en términos reduccionistas se tiende a creer, sino que implica -además- la calidad de la experiencia (Unión Internacional de Telecomunicaciones, 2002). Variados criterios clasificatorios que configuran una diversidad de tipología de usuarios; asumiendo que lo característico del usuario es el establecimiento de un tipo de relación práctica con la máquina, aunque no esté necesariamente interesado en la tecnología, excepto como algo que permite una aplicación (Turkle, 1999), se entenderá como tal aquella persona que utiliza un programa o hardware informático de cualquier naturaleza y propósito, dentro de una fracción de tiempo y con una intensidad determinada. Los usuarios serían, en consecuencia, personas o entidades que acceden a los contenidos que les proporciona la sociedad de la información a través de los medios que posibilitan dicho acceso (Cela, 2005).

Pero este aprovechamiento o uso de las Tecnologías de la Información y la Comunicación (TIC) se funda en condiciones sociales e individuales, tanto de carácter objetivo como subjetivo, que en modo alguno son equivalentes y aplicables a toda la población. La desigual distribución de condiciones sociales y capacidades personales implica niveles de acceso y aprovechamiento diferenciado de las bondades de la tecnología, que de paso configura una nueva y grave fractura social entre las personas que ya se comunican y coordinan actividades mediante redes digitales, de quienes aún no han alcanzado este estado avanzado de desarrollo (Tello, 2008).

Las altas tasas de crecimiento de Internet que se constatan en gran parte del globo, a excepción del África subsahariana, permiten estimar que, en el corto plazo, los núcleos centrales de los países en desarrollo estarán cada vez más conectados. No obstante lo alentador de este pronóstico, se debe ser consciente de que la conexión al interior de estos espacios no será homogénea, constatándose la existencia de territorios y segmentos de inclusión y exclusión. Por tratarse de un problema vinculado a procesos de revolución tecnológica no resulta del todo nuevo sino la extensión de una problemática que ha precisado ser reconceptualizada con base en el contexto histórico (Vega, 2007).

La complejidad política, económica y cultural de la desigualdad tiende a ser de tal magnitud que la brecha digital se constituye como una más que viene a profundizar las preexistentes, por lo que su abordaje en la política pública deberá encuadrarse en el conjunto de cuestiones de largo alcance que atañen a la desigualdad como un hecho estructural con implicaciones políticas y sociales relativas al orden mundial emergente (Bouza, 2003). Según el World Resources Institute (2000), estar a uno u otro lado de esta brecha digital significa tener diferenciadas oportunidades para tomar parte en la nueva economía sustentada en la información, donde cada vez más empleos están relacionados con las computadoras. Ubicarse en el lado menos aventajado significa tener menos oportunidades para participar de la educación, la capacitación, las compras, el entretenimiento y las oportunidades de comunicación disponibles en línea. Estas posturas son refrendadas por Norris (2001), quien afirma que las TIC incuban la promesa de mayor productividad y un singular potencial participatorio. La divisoria digital, en consecuencia, sea entre naciones (brecha global), entre personas (brecha social) o incluso entre quienes usan el potencial de la Internet, o no, como medio para empoderarse y participar políticamente (brecha democrática), condicionaría el desarrollo potencial limitando el acceso a los beneficios que podrían surgir del uso de tecnología.

Desde este entendido, enfrentar la segmentación digital se constituye en un imperativo ético, social y económico a fin de emparejar el terreno permitiendo a los potenciales usuarios hacer usufructo de sus bondades y beneficios (Villanueva, 2006). Caso contrario, el retraso económico 
se hará sentir como consecuencia y efecto de una brecha digital agudizada, complejizando la integración y cohesión social al no facilitar el acceso a los colectivos sociales más desfavorecidos y de las zonas más alejadas, inhibiendo la creación de empleo, así como el desarrollo de procesos de educación permanente, cada vez más necesarios (Cela, 2005).

La existencia de una sociedad de la información, y por defecto la reducción o eliminación de la divisoria digital, pasa necesariamente por la existencia de una población alfabetizada en el uso de las tecnologías, en cuanto a consumidores y productores de bienes digitales. Sin una fuerza de trabajo formada en la cultura y tecnología digital no podrán desarrollarse las empresas y servicios asociados a la sociedad de la información (Blázquez, 2001).

En la medida que la brecha digital se constituye en un problema de acceso y calidad de la relación establecida con ella, en términos de producción y consumo de bienes y servicios digitales, es que la escuela se configura en un lugar y espacio propicio que puede democratizar el acceso de aquellos que precisarán de esta herramienta en el futuro, y el medio para instalar competencias digitales para un uso con valor agregado. Por ser los jóvenes la generación de recambio, la nueva fuerza de trabajo que en el futuro mediato se insertará en el mercado laboral y productivo, los gobiernos han acercado el uso de las tecnologías al espacio educativo por medio de planes y programas que contemplan la implementación y capacitación en el uso de redes en las escuelas. Si bien disponer de tecnología no garantiza un uso de calidad, los dispositivos digitales en sus distintas versiones se configuran como un lugar común en las escuelas. En las unidades educativas europeas la presencia de TIC es una realidad palpable y evidente que ha dejado de ser una barrera para el uso educativo de las mismas (Area, 2010). Disponer de terminales con acceso a Internet conecta a profesores y alumnos a las redes de información, comunicación y conocimiento global; permite, por efecto de la exposición, generar competencias, niveles de dominio y de apropiación cada vez más complejos y elevados, y convierte a las TIC en un instrumento facilitador del acto educativo. No obstante esto, los estudios señalan usos diferenciados por parte de la población escolar, con base en el sexo y condición socioeconómica, en especial en lo referente al tipo e intensidad de acceso a Internet, así como en el uso de dispositivos recreativos, como los juegos (Segura, 2009).

En consecuencia y con base en lo expuesto, se asume, por un lado, que el usuario hace servir una aplicación, programa o hardware informático de cualquier naturaleza con diferenciados propósitos, tiempos e intensidades. Por otro lado, este aprovechamiento de las TIC se funda en condiciones sociales, institucionales e individuales de carácter objetivo y subjetivo, en modo alguno equivalente y extensible a toda la población. Es por ello que se ha buscado caracterizar y cuantificar las brechas y segmentaciones desde tres perspectivas analíticas: 1) la brecha del acceso, determinada por las limitaciones en la disponibilidad material a las tecnologías (computadores, redes e Internet), así como en la calidad de los mismos. Accesibilidad que, como expresión de atributos sociodemográficos, es de carácter público o privado; 2) la brecha de las capacidades individuales, que explora la desigual habilidad para usar las tecnologías como expresión de la precariedad del entorno cultural y social, así como de factores subjetivos como la autoconfianza. Situación que está mediada, entre otros factores, por el tiempo en habitar la red; y 3) la brecha de capital cultural y social, que hace alusión a la red como prolongación del espacio social, el cual está fuertemente influenciado por las posiciones sociales que condicionan el tipo y calidad de la circulación, así como el rol y privilegios que de ella se derivan como expresión de los usos y niveles de apropiación en la red. Accesibilidad, capacidades y capital cultural y social que configuran y condicionan tipologías diferenciadas de usuarios.

Para cada uno de estos niveles de análisis de la segmentación de los medios tecnológicos se realiza una caracterización tipológica en torno a los principales atributos que los definen y diferencian, en cuanto a tenencia, disponibilidad, nivel de experticia y conocimiento; además de las modalidades e intensidades en el consumo de tecnología, sus usos y aplicaciones que hacen servir y lugares de acceso. Ello tanto para los escolares usuarios de computadores como de Internet. 


\section{Método}

La investigación es de carácter cuantitativo de corte analítico, hace uso de base de datos pre existentes y tiene como propósito cuantificar los índices de penetración, conocimiento y uso que tiene y hace de las TIC la población estudiantil. Así como configurar la profundidad y dimensiones de la brecha digital al interior de este grupo. Como fuente de datos se utilizó la encuesta de Caracterización Socioeconómica Nacional (Casen) del año 2011, rescatando de allí el ítem "Patrimonio y Tecnología de Información y Comunicación" incorporado dentro del modo de "Residentes" de dicha encuesta. La Casen es una encuesta aplicada en el país por el Ministerio de Desarrollo Social de Chile y tiene por objeto testear el estado de la población respecto de sus condiciones de trabajo, salud, ingresos, educación y vivienda, constituyéndose en el principal instrumento de planificación de la política social en Chile. Esta encuesta se aplica en todo el territorio nacional a una muestra representativa de alrededor de 220,000 personas y 70,000 hogares, la cual es extraída mediante procedimientos de estratificación a fin de extrapolar resultados válidos a toda la población.

La unidad de análisis estaba constituida por el conjunto de estudiantes que a la fecha de aplicación de la encuesta se encontraban matriculados en algún establecimiento educacional público o privado, tanto de nivel básico (primaria) como nivel medio (secundario), con independencia de la modalidad científico humanista o técnico-profesional para el caso de la enseñanza media. El rango de edad iba de los 6 a los 18 años, extremos incluidos. Esto dio lugar a una muestra de 36,193 estudiantes encuestados, aunque para el análisis se ponderó por factor de expansión regional a fin de evitar el sesgo de selección y hacer los resultados extensivos y representativos a toda la población. La muestra estaba homogéneamente distribuida por sexo ( $50.6 \%$ varones) y el $87 \%$ provenía de zonas urbanas. En lo que se refiere a nivel y modalidad educacional $67.7 \%$ cursaba educación básica, un $25.8 \%$ la enseñanza media científico-humanista y un $6.5 \%$ técnico-profesional.

Dado que algunas de las preguntas del ítem "Patrimonio" dentro del modulo "Residentes" estaban condicionadas a la categoría de "Jefe de hogar" se utilizó la función "Agregar variable" con el fin de imputar las respuestas dadas por el jefe de familia al conjunto de estudiantes miembros del hogar. Ello con el propósito de hacer más representativa la información a esta unidad de análisis.

A través de tablas de contingencia se generó un contraste donde se explicitó la diferencia en la brecha digital de los estudiantes, reflejada ésta en la tenencia de tecnología, lugar de acceso, frecuencias y tipos de uso.

\section{Análisis de los datos}

\subsection{Incorporados y Excluidos}

Para constatar el nivel de acceso a computadora (PC) e Internet que presenta la población estudiantil en Chile se consultó a los jefes de hogar sobre la disponibilidad de dichos dispositivos al interior del espacio domiciliario. Esto en razón de que el simple acceso a las TIC ocupa en este segmento poblacional un espacio de cotidianidad cuyas diferencias se hacen sentir desde la perspectiva del lugar en que se materializa el acceso; se estima que quienes hacen servir estas aplicaciones desde contextos distintos del domiciliario evidencian accesos más restringidos, menos intensivos y menos frecuentes. El acceso desde el hogar no sólo posibilita usos más variados e intensivos de las tecnologías, sino que es mucho más significativo y relevante para las actividades de aprendizaje (Pedró, 2011).

En este sentido, los niveles de penetración de computadoras e Internet en el ámbito domiciliario han aumentado significativamente en la población escolar chilena dentro del período analizado. Si bien este nivel de accesibilidad no se equipara al logrado por otras TIC, como lo es el teléfono 
celular, el déficit de cobertura domiciliaria de PC e Internet se ha reducido en un 44 y $49 \%$, respectivamente. Así, por ejemplo, mientras en el año 2000 el 91.5\% de la población escolar señalaba no contar con Internet en casa, esta cifra se reduce al 51.3\% en el 2011. Cifra que si bien manifiesta una brecha significativa dentro del esquema dual del haves y have nots, no contempla la influencia de la disponibilidad de otros lugares de acceso como la escuela, centros comunitarios o lugares de pago que, especialmente en la población estudiantil, compensan la eventual ausencia de las TIC en el ámbito domiciliario y configura el Indicador de Acceso Universal, que al estar más centrado en la disponibilidad general que en la individual, reflejaría en forma más real el nivel de población cubierta por las tic. De esta forma, cuando se analiza el acceso con independencia del lugar, un alto porcentaje de los sujetos encuestados se declara usuario de Internet (83.6\%) alcanzando cuotas de cobertura muy por encima de la población en general (52.2\%). Si bien es un hecho comprobado que los jóvenes acceden con mayor facilidad a las TIC que los adultos, aun teniendo menor poder adquisitivo, (CEPAL, 2003) esta cifra debe analizarse con resguardos toda vez que no controla el tipo de acceso diferenciado en términos de calidad, intensidad, motivos y restricciones de uso.

Estos significativos niveles de acceso a las TIC e Internet por parte del conglomerado escolar se explica, por una parte, por la fuerte caída en los precios de las computadoras, terminales y redes, incluyendo el surgimiento de alternativas tecnológicas más baratas como los netbooks (Agostini y Willington, 2012); y por otra, por un factor subjetivo en cuanto al atractivo impacto que las tecnologías generan en la población juvenil. Para los jóvenes las TIC presentan más ventajas que peligros; les presenta un mundo lleno de posibilidades y contactos, del cual quieren formar parte. Para ellos estar conectados a este espacio interactivo, versátil y entretenido es la consigna. Favorable predisposición subjetiva que presiona a la familia y moviliza las opciones de compra y acceso a estos dispositivos, incluso a pesar de los reducidos ingresos (ver figura 1).

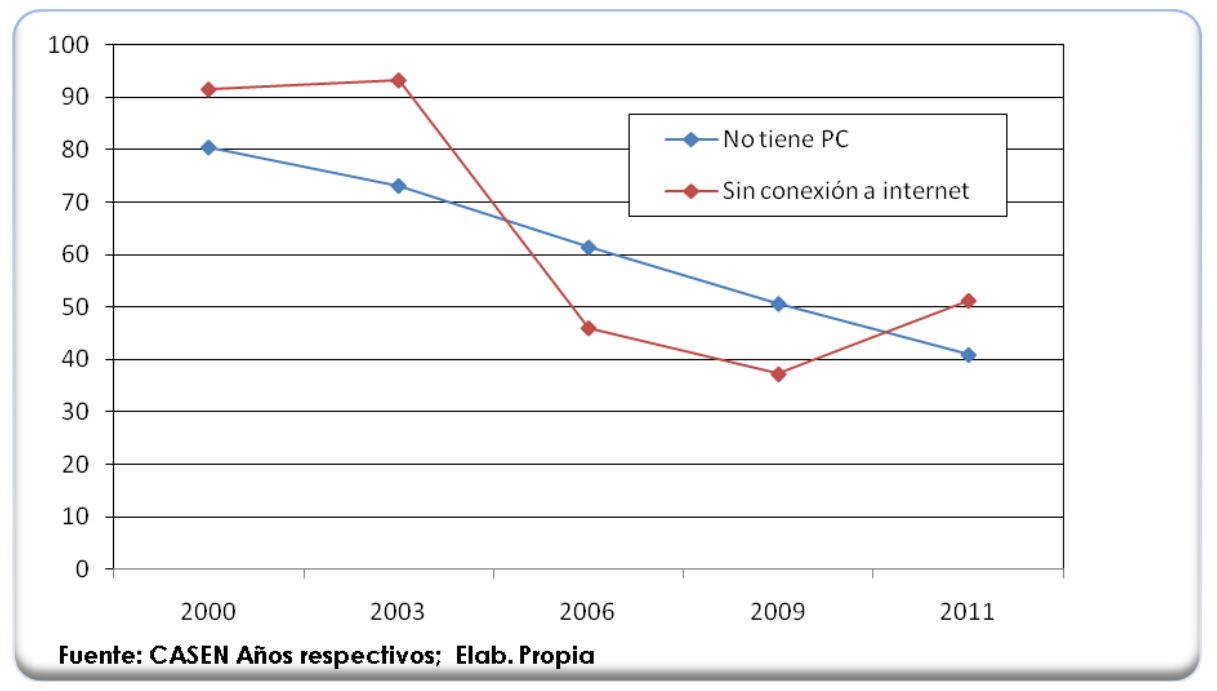

Figura 1. Evolución del Déficit de Cobertura en tenencia PC-Internet domiciliario (\%) Fuente: Casen años respectivos. Elaboración propia.

No obstante lo anterior, los usuarios en general, y la población escolar en particular, evidencian un vínculo más fluido y constante cuando la conexión se desarrolla en el contexto domiciliario, por lo que al considerar este elemento la brecha o proporción de excluidos de las tic se ensancha. Así entendido, al año 2011, cuando se consigna la existencia de Internet en el patrimonio familiar, encontramos que uno de cada dos estudiantes (51.3\%) señala no disponer de Internet, y el factor configurador de la brecha en términos de acceso es el nivel socioeconómico, evidencia consistente con los hallazgos nacionales e internacionales (Agostini y Willington, 2012; Jara, 2013; Pascual y Moder, 2013; Tello, 2008). No obstante, el desarrollo de políticas de aumento de 
cobertura de acceso universal, como el caso de los Infocentros en Chile, permitiría la democratización de las tecnologías sobre las barreras que imponen los ingresos familiares o la dispersión geográfica de la población, situación especialmente relevante cuando estadísticas nacionales e internacionales señalan que amplios sectores poblacionales usan ordenadores y accesan a Internet en espacios no domiciliarios, tales como centros de estudio, lugares de trabajo o centros de acceso comunitarios. Modalidad que, además, si bien tiende a democratizar el acceso y por medio de éste se constituye en uno de los factores explicativos de la penetración de Internet, da cuenta también de las condicionadas posibilidades de acceso y diferenciados tipos de uso. Modalidad de acceso extra-domiciliario que está fuertemente relacionado con factores socioeconómicos y étnicos de los grupos de usuarios; por eso no es casualidad que los estudios estadounidenses constaten que este tipo de acceso es mayoritario entre los miembros de las etnias hispana y afroamericana y de bajos niveles de renta, carentes de computadoras o conexión en el hogar.

Al considerar el lugar de acceso a Internet, se entra a un segundo tipo de brecha llamada doméstica o social, referida a aquellas diferencias en el acceso a una tecnología al interior de un país o de una comunidad (Fernández, 2005), observándose diferenciales niveles de aprovechamiento. frecuencias de uso. conocimiento y apropiación según el lugar de conexión. Ello supone disponer de mayores niveles de experticia por parte del usuario domiciliario al operar sin las desventajas que involucra el acceso extra-residencial, brecha fuertemente condicionada por el factor socioeconómico y capital cultural. Así se indica en la tabla I, conforme disminuyen los niveles de ingreso aumenta drásticamente la proporción de excluidos, especialmente relevante al comparar los quintiles extremos.

Tabla I. Niveles de exclusión digital según el quintil de ingreso

\begin{tabular}{l|c|c|c|c|c|c}
\cline { 2 - 5 } & \multicolumn{4}{c|}{ Quintil Autónomo Nacional } & \multirow{2}{*}{ Total } \\
\cline { 2 - 6 } & I & II & III & IV & V & \\
\hline Sin acceso a computador & 61.5 & 46.7 & 33.9 & 23.2 & 7.8 & 40.9 \\
Sin acceso a Internet & 76.6 & 58.8 & 44.3 & 28.2 & 8.1 & 51.3 \\
\hline
\end{tabular}

Fuente: Elaboración propia basada en Casen, 2011.

Para el primer quintil las tasas de exclusión en el acceso domiciliario a computadoras, y muy especialmente a Internet, son significativamente mayores en comparación con el quinto quintil, donde el riesgo relativo de exclusión en el acceso a Internet para los sectores sociales más precarios es 9 veces superior que el existente en los segmentos mejor posicionados en la escala de los ingresos, y 7 mayor en el caso de tenencia de PC. En consecuencia, los datos indican que una amplia mayoría de estudiantes de los estratos más vulnerables se encuentra limitada por carecer de dispositivos en sus hogares.

Los altos costos, de especial incidencia en los sectores sociales más postergados, se constituyen en una de las principales razones esgrimidas por las familias de los estudiantes a la hora de testear en los motivos para no conectarse a la red desde sus respectivos domicilios. En efecto, 8 de cada 10 estudiantes que no disponen de Internet señalan motivos de altos costos, cifra segmentada según nivel de ingresos. Un $73.1 \%$ en el primer quintil y un $46.5 \%$ en el quinto quintil señalan esta razón. De hecho, las razones asociadas al precio de Internet no sólo constituye un motivo para no contratar el servicio, sino también para suspenderlo (Subsecretaría de Telecomunicaciones de Chile [SUBTEL], 2009) (ver tabla II). 
Tabla II. Motivos para no tener Internet según el quintil de ingreso

\begin{tabular}{l|r|r|r|r|r|r}
\cline { 2 - 5 } & \multicolumn{1}{c|}{ Quintil Autónomo Nacional } & \multirow{2}{*}{ Total } \\
\cline { 2 - 5 } & \multicolumn{1}{c|}{ I } & \multicolumn{1}{c|}{ II } & \multicolumn{1}{c|}{ IV } & \multicolumn{1}{c}{ V } & \\
\hline Para evitar acoso a los niños & .4 & .8 & 1.2 & 1.6 & 3.7 & .8 \\
Para evitar uso de información personal & .2 & .3 & .6 & .4 & 1.2 & .4 \\
Por no saber utilizarla & 1.9 & 1.4 & .8 & 1.2 & 1.7 & 1.5 \\
No existir servicio & 4.4 & 5.1 & 5.2 & 7.2 & 8.1 & 5.1 \\
No interesarle & 17.4 & 18.5 & 20.4 & 26.5 & 25.2 & 19.1 \\
Ser demasiado caro & 73.1 & 70.6 & 66.7 & 56.2 & 46.5 & 69.4 \\
Accede por otro sistema & 2.6 & 3.3 & 5.1 & 7.0 & 13.7 & 3.8 \\
\hline
\end{tabular}

Fuente: Elaboración propia basada en Casen, 2011.

Estas razones de orientación económica están transversalmente presente en todos los segmentos sociales, pero con significativos matices respecto de la población en general. En este sector, si bien el alto precio del servicio es la principal razón para no tener acceso a Internet, siempre con mayor preponderancia en los quintiles más bajos, el factor económico no es en esencia el claro dominador, emergiendo aquí en importancia el desconocimiento de sus usos, la ignorancia respecto de la utilidad de Internet o el desinterés, similares hallazgos son constatados por Agostini y Willington (2012), motivos que en la población estudiantil tienen una importancia más reducida. En efecto, menor preponderancia registrarían las razones asociadas a la falta de interés o escasa valoración asignada a estas aplicaciones (19.1\%), en especial para los grupos familiares de mayores ingresos. Por otra parte, razones de privacidad y de seguridad -tales como resguardo de los datos de carácter personal o evitar situaciones de acoso a través de la red hacia los niños- tienen una presencia marginal en este segmento juvenil. En consecuencia, las razones están matizadas según el estadio del ciclo vital.

Para los jóvenes, que por lo general no perciben ingresos y dependen de las transferencias monetarias de sus padres, es lógico pensar que sea el motivo económico la principal dificultad para acceder a Internet, un espacio donde se mueven, a diferencia de sus progenitores, con naturalidad y despreocupación. A los padres, en cambio, les preocupa el impacto de las TIC en sus hijos, herramientas que a su juicio presentan luces y sombras. Por una parte, es una oportunidad para acceder a la información y conocimiento siendo una potente herramienta para apoyar el estudio, pero por otra es una amenaza, lo cual les hace asumir una actitud más protectora hacia sus hijos, en particular en lo referente a explotación sexual, acceso a pornografía. Otro aspecto valorado negativamente por los padres es que la red desarrollaría una baja capacidad a la frustración en los más jóvenes, dada la costumbre online de obtener gratificaciones instantáneas (Godoy y Galvez, 2010). Aspectos todos ellos que hacen que la red se mire con un dejo de desconfianza por la población adulta, especialmente por parte de los padres, como se observa en la figura 2. 


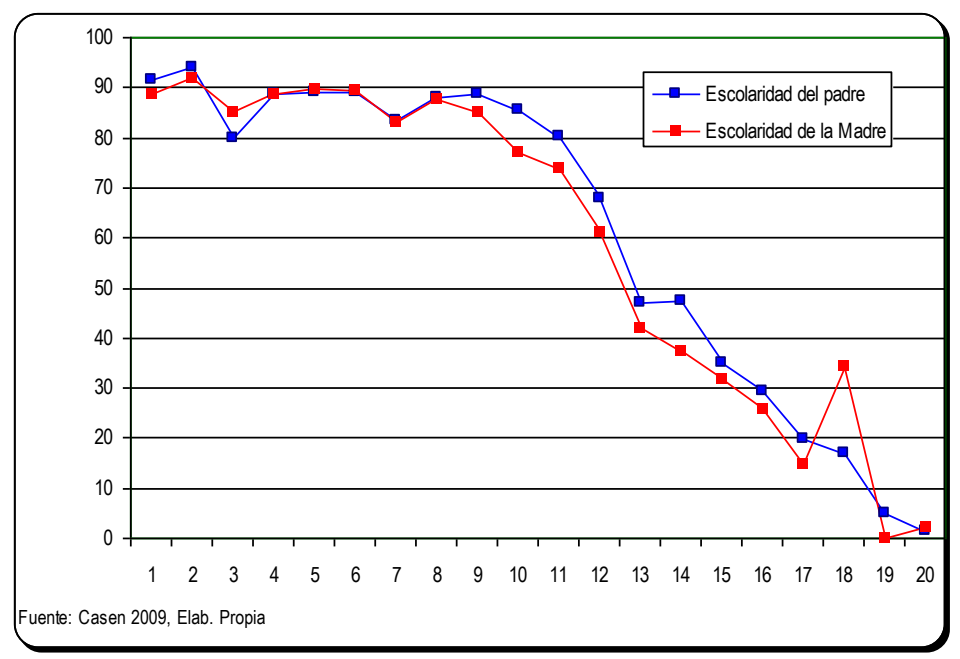

Figura 2. Índice de exclusión según Escolaridad de los Padres

Si bien el nivel de ingresos familiares recibe la influencia del capital humano acumulado de los progenitores, al contrastar los niveles de escolaridad de los padres con el índice de exclusión se observa una fuerte y significativa correlación, situación que se hace particularmente evidente a partir de los 9 años de escolaridad. A menor educación de los padres, mayor exclusión tecnológica de los hijos (SUBTEL, 2009). El capital humano acumulado, esencialmente cuando es de naturaleza avanzado, repercute tanto en las posibilidades de compra y de acceso a servicios de pago de Internet debido a mayores ingresos, como también a la mayor valoración que los padres tienden a atribuirle a las potencialidades y ventajas que representa el estar conectado, en especial cuando se tiene hijos en edad escolar; a diferencia de aquellos grupos familiares de menores ingresos en los que las prioridades son otras (Pascual y Moder, 2013). Los hijos, además, son entes dinamizadores de la interacción familiar en lo que a tecnología en el hogar se refiere, son un motivador que incluso ejerce presión sobre los progenitores para la adquisición y uso de TIC. Amparados en su mayor conocimiento tecnológico dentro de las familias, proporcionan acceso indirecto a Internet a sus padres, transfieriendo de manera informal conocimiento y destrezas en el uso de la herramienta, aunque se dé con impaciencia y poco sentido pedagógico (Godoy y Galvez, 2011). Conocimiento que eventualmente modificará en los progenitores el perfil actitudinal y aprensión hacia la tecnología. Mayor valoración parental de las bondades de las tecnologías que posibilitará que los padres inviertan, estimulen y brinden las condiciones para su uso y apropiación con sentido, situaciones que marcan brechas significativas entre los estudiantes que poseen estas condiciones y los que carecen de ellas (Burgos, 2012).

El problema del acceso, en consecuencia, no se resuelve con disponer del aparato en casa, sino más bien de la condición de hacerlo socialmente necesario para poder utilizarlo y obtener de ellas adecuados niveles de aprovechamiento, lo cual dependerá de las condiciones de existencia y reproducción del capital cultural de cada grupo (Ford, 1999), el cual se encuentra desigualmente distribuido incluso en la población estudiantil universitaria, a pesar de la homogeneidad que es posible suponer en instituciones académicamente selectivas (Roco, 2007). Capital cultural incorporado que ahora se constituye en uno de los elementos de mayor fuerza explicativa en el modelo de apropiación tecnológica, restando importancia a dimensiones tales como el acceso (Jara, 2013). Dicho en otros términos, disponer de infraestructura y redes puede entenderse apenas como el primer paso o entrada al acceso igualitario (Crovi, 2008; Sánchez, Olivares y Alvarado, 2013), pero si éste no supera la concepción reduccionista de simple herramienta y no son aplicadas apropiadamente y en el contexto cultural adecuado, su impacto en la reducción de la brecha digital será mínimo (Serrano y Martínez, 2003). 


\subsection{Segmentación de los lugares de acceso}

Existe una gama de lugares de acceso. Sus tipos transitan desde el ámbito domiciliario o doméstico a los de acceso público, sean de pago o gratuitos, pasando por las redes sociales de apoyo constituidas por amigos, familiares o conocidos, para finalizar en el centro educativo, pero a pesar de su variedad, las posibilidades de uso están fuertemente segmentadas por el factor socioeconómico (ver tabla III).

Tabla III. Lugar de Acceso Internet según Nivel Socioeconómico

\begin{tabular}{|c|c|c|c|c|c|c|}
\hline & \multicolumn{6}{|c|}{ Quintil Autónomo Nacional } \\
\hline & I & II & III & IV & $\mathrm{V}$ & Total \\
\hline En el Hogar & 24.6 & 41.1 & 55.5 & 70.6 & 90.3 & 48.6 \\
\hline En el Trabajo & 0.3 & 0.3 & 0.8 & 0.5 & 0.5 & 0.5 \\
\hline En el Establecimiento educacional & 31.4 & 23.5 & 17.6 & 11.1 & 4.1 & 20.8 \\
\hline En un Telecentro comunitario o Infocentro & 0.7 & 0.8 & 0.3 & 0.4 & 0.2 & 0.5 \\
\hline En un lugar de pago (cybercafé) & 15.2 & 15.4 & 10.9 & 6.5 & 1.1 & 11.6 \\
\hline En otro lugar & 2.0 & 1.8 & 1.5 & 1.4 & 0.6 & 1.6 \\
\hline
\end{tabular}

Fuente: Elaboración propia basada en Casen, 2011.

El espacio domiciliario es el claro dominador al momento de definir el lugar de acceso a Internet (48.6\%). La escuela ocupa un lugar secundario y muy por debajo del hogar, aunque su importancia se hace relevante conforme disminuyen los niveles de ingreso familiar. La escuela, a diferencia de los hogares, se constituye en el gran democratizador del acceso a las TIC, potenciando las posibilidades de adquisición de mayor capital social y cultural por parte de los estudiantes (Sunkel, 2006), aunque estas posibilidades se encuentren limitadas por las restricciones propias del espacio educativo, situación que limita los niveles de acceso y apropiación de los usuarios. Así entendida, la brecha digital se expresa en una escala de segmentos sucesivos según niveles de apropiación y uso que transitan más allá del simple acceso que configura el tener/no tener, esterilizando o limitando las posibilidades de aprovechar los beneficios que ofrecerían las tecnologías. Segmentación sociotecnológica que ensancha el concepto de brecha más allá de los procesos de digitalización, abarcando la ciencia, el conocimiento intuitivo o no organizado, la educación, la cultura; es decir, lo que Bourdieu (2002) llamó "capital cultural".

El lugar de uso de Internet es relevante pues condiciona fuertemente el tipo de actividades que los usuarios pueden realizar, así como la frecuencia de acceso y tiempo que destinan a ello. Concordante con esto es que el lugar de uso preferido por los usuarios son las residencias particulares cuando en ellas disponen de Internet (96.4\%). Predilección basada en que hay mayor tranquilidad, seguridad y resguardo a la intimidad, atributos altamente valorados por los estudiantes usuarios de tecnología, aspectos que los espacios extra-domiciliarios no resguardan de forma adecuada, razón por la cual el uso en estos últimos no sólo es mucho menor sino que además se observa una disminución en su importancia relativa (Agostini y Willington, 2012).

Frente a la ausencia de conexión domiciliaria en la red, le siguen en importancia la Escuela y los lugares de pago (53.6\% y 31.3\% respectivamente). Lugares de acceso también fuertemente condicionados por el nivel socioeconómico de los estudiantes, pero cuyo uso por parte de los alumnos de los sectores más marginados se ve posibilitado por la alta penetración que estos dispositivos tecnológicos registran en la escuela. Es en los centros educativos, como lugar de acceso y a pesar de sus dificultades, donde se constata una conexión más masiva y democrática. Y ellos están por encima de lo que es posible esperar en el hogar, pues si bien el hogar es el lugar de acceso preferido por los usuarios, al estar condicionado a la tenencia de computadora e Internet en casa (cuya penetración está fuertemente segmentada socialmente) su importancia se relativiza al momento de considerar a la población en general. La relevancia de la escuela a este respecto es expresión de la focalización de las políticas públicas de acceso a Internet que 
fomenta la presencia de lugares públicos o gratuitos como los establecimientos educacionales para brindar oportunidad de uso a quienes no tienen una suscripción en el hogar (Agostini y Willington, 2012).

Cuando la habitualidad del lugar de uso está condicionada por factores socioeconómicos, la escuela se configura como un elemento de trascendental importancia para garantizar al acceso a una porción importante de los estudiantes, en especial de los que pertenecen a los quintiles de menores ingresos, contribuyendo de paso a la reducción de las brechas existentes. El $58.5 \%$ del $20 \%$ más pobre de la población estudiantil que no tiene conexión a Internet señala conectarse frecuentemente desde el establecimiento educacional. La escuela, no obstante sus limitaciones y dificultades, cumple un importante rol compensatorio de las deficiencias del entorno social inmediato de este grupo, atenuando la brecha digital. Más aún, cuando se constata la debilidad de las redes sociales que, a diferencia de los otros estratos, no se perfila como un ente que posibilite el acceso a la red para este segmento estudiantil. La segmentación socioeducativa no sólo divide y heterogeniza el espacio escolar, sino que también homogeniza hacia el interior de la escuela o intra-grupo, por lo que las redes sociales de apoyo de los sectores más vulnerables son igual de precarias en el acceso que el sujeto demandante.

En cuanto a las escuelas como lugares de uso es importante señalar dos conclusiones de relevancia. Primero, en su aspecto positivo se pone de manifiesto la importancia que revisten los establecimientos educacionales para garantizar el acceso a la población más vulnerable del país. Grupo que de no mediar la existencia de estos centros registrarían fuertes dificultades y restricciones para integrarse a la red y pasarían a engrosar la brecha de los excluidos de la Sociedad de la Información. Es decir, la escuela opera como bisagra para el acceso a los sectores socioeconómicamente menos favorecidos y atenúa la brecha digital, pues representa la principal fuente de acceso gratuito a las TIC.

Por otro lado, la escuela se activa como posibilidad relevante de uso sólo en la medida que los ingresos están por debajo de determinado nivel crítico que inviabiliza las otras alternativas percibidas como más atractivas por los usuarios, como sería la conexión domiciliaria y los lugares de pago. Esto constata la presencia de barreras de naturaleza administrativa o de infraestructura instalada en la escuela que inhiben su uso. A este respecto, es importante recordar que lo que caracteriza a los usuarios de centro de pago (cybercafé) es, además de no disponer de Internet en casa, el hecho de valorar significativamente la privacidad, seguridad y velocidad de los que disponen en estos lugares. Características que en teoría la escuela no brindaría. En este mismo sentido, el principal atributo valorado por los usuarios de Internet en los colegios, especialmente de los quintiles más pobres, es que es gratuito y no disponen de otro lugar. Por lo tanto, en la medida que el uso de estos centros está en función de los ingresos precarios, es lógico suponer que mejorando dicho nivel socioeconómico cambiarán también las preferencias de los usuarios. Ya a este respecto, el World Internet Project [WIP] (2006) encontró que el único lugar de uso que ha presentado una leve tendencia hacia la baja entre los años 2003 y 2006 son, precisamente, los establecimientos educacionales. El abaratamiento de los costos de acceso y la mejora en los ingresos estarían actuando como factores explicativos.

Lo cierto es que el acceso no es unimodal. A pesar de los evidentes matices referentes a los lugares de uso de computadoras e Internet, así como al hecho de que es la residencia particular el lugar de acceso predilecto a las TIC por parte de los estudiantes, se constata también el uso en paralelo de los establecimientos educacionales y de los cybercafés, a los que les corresponde una función subsidiaria al hogar, no obstante la heterogénea adhesión por parte de los usuarios condicionada por los niveles de renta. 


\subsection{Segmentación de los usos}

Las TIC no son sólo equipamiento, su omnipresencia en todas las esferas del quehacer social dados sus altos niveles de penetración en el trabajo, el hogar, los servicios, la administración, el comercio y la educación, impregnan a estos aparatos de una serie de significados asociados a su utilización que trasciende el mero fin utilitario explícito. Las tecnologías son expresión viviente de la promesa del desarrollo personal construido en la interacción con otros sujetos virtuales, contenidos y servicios electrónicos en un contexto de absoluta libertad. Superadas las barreras temporo-espaciales, la libertad es un principio fundamental. Libertad expresada en la posibilidad ilimitada de elegir cuándo, dónde, con quién efectuar las funciones cotidianas, a qué contenidos exponerse y qué servicios elegir.

Pero este gran resort virtual del all inclusive opera a escala planetaria, pero no mundial. No todos, por diversas razones, pueden pagar el peaje de estas autopistas de la información. Investigaciones como la de WIP (2005) señalan que, no obstante las importantes reservas de usuarios potenciales, siempre en un contexto de pleno acceso, la penetración de determinadas tecnologías, como Internet, está llegando a una etapa de crecimiento desacelerado. La era de la información opera con una lógica de integración y exclusión. Como corolario de esto, quienes no acceden a esta red de flujo se perfilan como desadaptados y excluidos. Habitantes de la frontera en la era del acceso cuyo actuar en este nuevo mundo, en términos eficientes y efectivos, se ven claramente comprometidos, cuando no imposibilitados. Son los analfabetos de la posmodernidad, imposibilitados de entender estos nuevos códigos y de manejarse en este planeta interconectado y complejo, donde amenazas y oportunidades están a un clic.

Pero el problema no se traduce exclusivamente al simple acceso, es la puerta de entrada para incrementar la cantidad de usuarios y por medio de esto la disponibilidad de contenidos y servicios. Esta exclusión o invisibilidad de importantes sectores está condicionada a factores objetivos, como infraestructura instalada y accesible, pero también a condicionantes subjetivas. La percepción de ser parte y tener capacidades que posibiliten una incorporación con sentido se ve expresada en los servicios y contenidos que se demandan de la red. Niveles de apropiación y usos con sentido claramente segmentado por componentes económico-sociales. Diferencias que se hacen sentir al interior de cada conglomerado territorial y grupos sociales (ver tabla IV).

Tabla IV. Modalidad de uso según Nivel Socioeconómico

\begin{tabular}{l|r|r|r|r|r|r}
\cline { 2 - 6 } & \multicolumn{1}{c}{ Quintil Autónomo Nacional } & \multirow{2}{*}{ Total } \\
\cline { 2 - 6 } & 95.5 & 94.9 & 94.0 & 94.4 & 95.6 & 94.9 \\
\hline Obtener información & 71.1 & 75.4 & 80.3 & 84.6 & 87.3 & 78.2 \\
Comunicación escrita & 22.2 & 23.3 & 26.7 & 31.8 & 46.6 & 28.0 \\
Comunicación por voz & 80.3 & 83.2 & 87.7 & 91.3 & 94.5 & 86.0 \\
Entretenimiento & 5.9 & 7.7 & 9.2 & 12.4 & 15.1 & 9.2 \\
Comercio electrónico & 1.3 & 1.3 & 1.9 & 3.7 & 4.4 & 2.2 \\
\hline Operaciones de banca electrónica
\end{tabular}

Fuente: Elaboración propia basada en Casen, 2011.

Así, de los 10 usos más populares de Internet, Chile y Estados Unidos coinciden en seis, entre ellos tener e-mail, navegar, leer noticias y jugar. Pero mientras los usuarios estadounidenses agregan a su consumo digital las compras, hacer reservas de viaje, buscar información médica y revisar cuentas bancarias, el internauta chileno opta por usarlas para estudiar, bajar música, revisar avisos clasificados y chatear. Es así como, a nivel general, el uso de contenidos y servicios que con mayor frecuencia utilizan los estudiantes tienen relación con la búsqueda de información de cualquier naturaleza, incluida aquella para apoyar o desarrollar actividades educativas (94.9\%), entretenimiento (86\%) y comunicación escrita (78.2\%). Usos informativos, comunicativos y de entretenimiento que son expresión común al indagar el consumo de Internet, el cual se constituye en uno de los medios preferidos por los usuarios chilenos para comunicarse con otras personas y pasar el tiempo, restando la otrora hegemonía que ocupaban el teléfono 
(comunicarse) y la televisión (pasatiempo). Patrón que evidencia mayor intensidad en los jóvenes, donde incluso las brechas por segmento socioeconómico se hacen más tenues.

Asimismo, se constata la utilización de otras modalidades de uso con presencia mucho más marginal, tales como operaciones con banca electrónica (2.2\%), comercio electrónico (9.2\%). Actividades de carácter económico-productivo que pierde relevancia al compararse con aquellas vinculadas a la comunicación y entretenimiento, y se distancia del patrón de uso que hace de ella la población adulta. No obstante el uso esporádico que los jóvenes hacen de estas aplicaciones, es precisamente en ellas donde se manifiestan las mayores brechas con base en el perfil socioeconómico del usuario al precisar otro tipo de competencias.

Que las diferencias de status social estén presentes en la utilización de servicios de corte o finalidad comunicativa online, mas no en el uso de servicios informativos, estaría asociado a las exigencias particulares que se demandan de los servicios en cuanto a tiempo, asequibilidad, redes sociales y condiciones infraestructurales. Las aplicaciones de uso comunicativo y de entretenimiento exigen, con frecuencia, un contacto más prolongado en el tiempo, en especial cuando este contacto es en tiempo real, lo que implica un mayor coste en tiempo y dinero, no siempre posible de solventar cuando el acceso se hace desde un centro de pago o colegio, lugares preferenciales de acceso de los grupos sociales más precarios.

Por otra parte, dentro de las actividades de perfil comunicativo de uso menos frecuente por los estudiantes está la comunicación por voz (28\%), la que a su vez observa una mayor segmentación socioeconómica. Mientras en el quintil de mayores ingresos un $46.6 \%$ señalaba utilizar esta aplicación, esta cifra se reduce a tan solo un $22.2 \%$ en el primer quintil. Dada la homogeneidad de las redes sociales de los estratos de menores ingresos, que se movilizan en la misma precariedad infraestructural, difícilmente podrán los usuarios más vulnerables utilizar este medio рага establecer contacto con sus pares con la misma fluidez, rapidez y calidad de respuesta como lo hacen los de sectores socialmente más aventajados. Estos últimos se conectan desde sus casas, con ancho de banda que permiten fluidez a la comunicación por voz, obviando así las restricciones del espacio escolar donde este tipo de uso está limitado o proscrito.

No obstante lo anterior, los usos de comunicación escrita y voz son recursos muy extendido entre los jóvenes, y se constituyen en una pieza fundamental en el cambio tecnológico que experimentamos. Componente que hace distanciarse drásticamente del simplista modelo informacional como principal atributo de la red. Usos que se extienden a pesar de la valoración negativa que tienen de ella especialmente en la escuela en tanto la consideran una pérdida de tiempo, una utilización indeseable de la red que se presta a un uso poco decoroso de las comunicaciones. Se suma a ello la impresión de que su consumo excesivo es fuente de trastornos de personalidad y termina por aislar a las personas, destruyendo sus vínculos sociales. Estigmatización tecnofóbica de las TIC, expresión de temores aún muy arraigados y que persisten a pesar de que la evidencia disponible apunta a desmitificar esta creencia por cuanto las TIC se integran a la vida cotidiana reforzando hábitos y vínculos sociales preexistentes. La red, con sus luces y sombras, es también extensión representativa del mundo físico y real reproduciendo lo que acontece en éste con mayor inmediatez, resolución e intensidad, generando así para los jóvenes una suerte de realidad aumentada, un espacio pletórico de posibilidades del cual se busca ser parte y ampliar así la visión que se tiene del mundo (PNUD, 2006) a pesar de los peligros eventuales.

La red, a pesar de haberse posicionado rápidamente como alternativa de recreación y comunicación, siendo estos los servicios más demandados de Internet; es todavía un canal marginal para el comercio, el consumo y la participación ciudadana. Comportamiento de los usuarios que, si bien está explicada por la misma configuración etárea de la población, nos distancia fuertemente de los exhibidos por sociedades más desarrolladas. El comercio electrónico es todavía una tarea pendiente en la sociedad chilena, escasa aplicación de la que no es ajena la población escolar consultada. Estudios que han procurado abordar la explicación a la escasa agresividad transaccional en la modalidad electrónica del usuario nacional se relaciona con el 
poco tiempo que llevan conectados a la red. Otras razones relevantes son de tipo cultural, pues el consumidor promedio valora positivamente el "vitrineo" en centros comerciales, a lo cual se suma la creciente aprensión que genera la seguridad de los datos personales. Dicho de otro modo, existiría una mezcla de razones, positivas y negativas, para distanciarse de la práctica del comercio y administración electrónica. Las primeras, amparadas en razones culturales, se relacionan con la preferencia por canales más formales y tradicionales. La negativa se asocia con el temor a dar información en la red, y al desconocimiento de Internet y de las plataformas o sitios en particular. Temores que se configuran en los principales motivos inhibitorios del comercio electrónico en Chile y el mundo, aunque se han atenuado con el paso del tiempo.

La incorporación de las TIC en el espacio educativo buscaba abrir las puertas de esta institución al desarrollo tecnológico y sus fuentes de información y conocimiento, de manera tal que posibilite cambios en los modelos educativos, de gestión escolar y en la didáctica de la enseñanza. Pero esta es todavía una transformación en espera, prueba de ello es que el uso que se hace de las TIC para propósitos de educación y capacitación es más bien marginal. Pareciera ser que el gran logro de la irrupción de las computadoras en la escuela ha sido brindar acceso a la tecnología a sectores sociales que de otra manera no podrían aprovechar las oportunidades que la sociedad de la información brinda. Es decir, su incorporación estaría más relacionada con el acceso equitativo a las tecnologías que con su uso pedagógico o educativo, como era su intención primaria. Lo anterior se constata por los bajos niveles de uso que tiene la herramienta para fines educacionales o de capacitación, no obstante recibir la influencia del sector socioeconómico de pertenencia. Quienes más hacen uso de las computadoras en la escuela, y a pesar de las dificultades administrativas y burocráticas, son los pertenecientes a los quintiles de menores ingresos.

\section{Conclusiones}

- Si bien la tenencia y acceso a PC e internet han aumentado significativamente en la última década, el factor socioeconómico sigue siendo determinante en la constitución de la brecha digital, que no se expresa únicamente por las posibilidades de acceso a las TIC, sino también en los diferenciados niveles de uso, conocimiento y aprovechamiento de las oportunidades que éstas ofrecen en los ámbitos de la comunicación, el entretenimiento, la información y el consumo.

- El capital socioeconómico y cultural que tienen las familias de origen del estudiante marcan diferencias en la variedad y profundidad de la penetración tecnológica en el hogar, y segmenta el uso que se hace de las TIC en intensidad, modalidad y frecuencia.

- Si bien uno de los principales objetivos de la masiva incorporación de las TIC en los establecimientos educacionales era el desarrollo tecnológico y la creación de estrategias que optimizaran el aprendizaje de los estudiantes, esta utilización con fines de capacitación y educación son limitadas.

- El establecimiento educacional se configura como el principal elemento compensador en lo que se refiere al acceso a las TIC e Internet para los estudiantes provenientes de los sectores de menores ingresos. No obstante, aquellos que acceden a la red desde la escuela presentan niveles de aprovechamiento y uso diferenciado respecto de quienes cuentan con conexión domiciliaria. Es en el espacio domiciliario donde los usos son más intensivos y variados, pues no presentan las restricciones infraestructurales y orgánico administrativa de la escuela.

- En cuanto aumenta la penetración de las tic dentro de un sistema social, los segmentos de población con el estatus socioeconómico más alto tienen tendencia a adquirir la información más rápidamente que los estratos de nivel socioeconómico más bajo, así que el desnivel de conocimiento y uso entre los segmentos tiende a aumentar. Los sectores 
de bajos ingresos y menor nivel educativo tienden a hacer un uso más simple de internet, accediendo eventualmente a contenidos menos complejos y avanzados. El problema además se traduce en que, en la medida que son los usuarios los que definen el tipo de aplicación y el curso del desarrollo de la tecnología, los rezagados y marginados tendrán cada vez menos que decir al respecto.

\section{Referencias}

Agostini, C. y Willington. M. (2012). Acceso y uso de internet en Chile: evolución y factores determinantes. Persona y sociedad, 26(1), 11-42.

Area, M. (2010). El proceso de integración y uso pedagógico de las TIC en los centros educativos. Un estudio de casos. Revista Educación, 10(5), 77-97.

Blázquez, F. (2001). Sociedad de la información y educación. México: Consejería de Educación, Ciencia y Tecnología.

Bouza, F. (2003). Tendencias a la desigualdad en Internet: la brecha digital (digital divide) en España. Madrid: Sistema.

Bourdieu, P. (2002). La distinción. Criterios y bases sociales del gusto. México: Taurus.

Burgos, E. (2012). Influencia del capital cultural mediático en las competencias comunicacionales de los bachilleres varguenses. Temas de Comunicación, 24, 157-187. Recuperado de http://revistasenlinea.saber.ucab.edu.ve/temas/index.php/temas/article/viewFile/743/717

Cela, J. (2005). Sociedad del conocimiento y sociedad global de la información: Implantación y desarrollo en España. Documentación de las Ciencias de la Información, 28, 147-158. Recuperado de http://revistas.ucm.es/inf/02104210/articulos/DCIN0505110147A.PDF

Comisión Económica para América Latina y el Caribe (2003). Los caminos hacia una sociedad de la información en América Latina y el Caribe. Santiago de Chile: ONu.

Fernández, F. (2005). Brecha e inclusión digital en Chile: Los desafíos de una nueva alfabetización. Comunicar: Revista Científica de Comunicación y Educación, 24, 77-84.

Ford, A. (1999). La marca de la bestia. Identificación. desigualdades e infoentretenimiento en la sociedad contemporánea. Buenos Aires: Norma.

Godoy, S. y Galvez, M. (2011). La brecha digital correspondiente: obstáculos y facilitadores del uso de TIC en padres de clase media y media baja en Chile. Revista Iberoamericana de Ciencia, Tecnología y Sociedad, 6(18). Recuperado de http://www.revistacts.net/volumen-6-numero18/103-dossier/407-la-brecha-digital-correspondiente-obstaculos-y-facilitadores-del-uso-de-ticsen-padres-de-clase-media-y-media-baja-en-chile

Jara, I. (2013). Desarrollo de habilidades digitales para el siglo XXI: ¿Qué nos dice el SIMCE TIC? Santiago de Chile: LOM.

Jara, I. (2009). Impacto de las TIC en el sistema educativo chileno. Centro de Estudios de Políticas y Prácticas en la Educación. Recuperado de http://mapeal.cippec.org/wp-content/uploads/2014/ 05/Impacto-de-las-TIC-en-el-sistema-educativo-chileno.pdf

Norris, P. (2001). Digital divide: civic engagement. Information poverty and the Internet worldwide. Cambridge University press. 
Pascual, J. y Moder, M. (2013). Desarrollo de habilidades digitales para el siglo XXI en Chile: ¿Qué nos dice el SIMCE TIC? Santiago de Chile: LOM.

Pedró, F. (2011). Tecnología y escuela lo que funciona y por qué. Recuperado de http://www.fundacionsantillana.com/upload/ficheros/noticias/201111/documento bsico.pdf

Programa de las Naciones Unidas para el Desarrollo. (2006). Guía para la transversalización de género. Santiago de Chile.

Roco, R. (2007). Mirando entre los intersticios de la brecha digital en la Educación Superior. Revista Calidad en la Educación, 26.

Sánchez. J. Olivares. R. y Alvarado. P. (2013). Variables asociadas al hogar y resultados de la prueba SIMCE TIC. En Desarrollo de habilidades para el siglo XXI: ¿Qué dice el SIMCE TIC? Santiago de Chile: LOM.

Segura, M. (2009). Panorama internacional de las TIC en la educación: barreras actuales y propuestas de futuro. Telos: Cuadernos de comunicación e innovación, 78. Recuperado de https://telos.fundaciontelefonica.com/telos/articulocuaderno.asp@idarticulo=3\&rev=78.htm

Serrano, A. y Martínez, E. (2003). La brecha digital: mitos y realidades. México: Editorial Universitaria.

Subsecretaría de Telecomunicaciones. (2009). Encuesta sobre Acceso. Uso y Usuarios de Internet Banda Ancha en Chile. Santiago de Chile: Autor.

Sunkel, G. (2006). Las Tecnologías de la Información y la Comunicación (TIC) en América Latina: Una exploración de Indicadores. Santiago de Chile: Serie Políticas Sociales.

Tello, E. (2008). Las Tecnologías de la Información y Comunicaciones (TIC) y la brecha digital. Revista de Universidad y Sociedad del Conocimiento, 4(2). Recuperado de http://www.uoc.edu/rusc/4/2/dt/esp/tello.html

Turkle, S. (1999). Cyberspace and identity. Contemporany Sociology, 28(6), 643-648. Recuperado de http://people.exeter.ac.uk/ip501/Turkle-Cyberspaceldentity.pdf

Unión Internacional de Telecomunicaciones. (2002). Informe sobre el Desarrollo Mundial de las Telecomunicaciones. Santiago de Chile: Autor.

Vega, R. (2007). Brecha digital: un problema multidimensional de la sociedad emergente. Inclusão Social, 2(2), 96-108. Recuperado de http://revista.ibict.br/inclusao/article/viewFile/1605/1811

Villanueva, E. (2006). Brecha digital: descartando un término equívoco. Razón y Palabra, 51. Recuperado de http://www.razonypalabra.org.mx/anteriores/n51/evillanueva.html 